\title{
Penetration and bouncing during impact in shallow cornstarch suspensions
}

\author{
Ricardo Arturo Lopez-de-la-Cruz ${ }^{1}$ (i) $\cdot$ Devaraj van der Meer ${ }^{1}\left[\right.$ [ $\cdot$ Adeline Pons $^{1,2}$ (])
}

Received: 28 February 2019 / Published online: 8 January 2020

(c) The Author(s) 2020

\begin{abstract}
The impact-activated solidification of cornstarch suspensions has proven to be a multi-faceted problem and a complete explanation of the different phenomena observed during this process remains elusive. In this work, we revisit this rich problem and focus on impact on shallow suspension baths where the solidification partly leads to bouncing of the impactor. We systematically vary the depth and solid fraction of the suspension, the mass of the impactor, and the impact velocity to determine which conditions lead to bouncing. For cases where bouncing occurs we observe distinctly different dynamics as compared to those cases without it. Our results allow us to connect the velocity oscillations and stop-go cycles that were observed during settling in a deep layer, with more recent work dealing with high-force and high-speed impact on a cornstarch suspension.
\end{abstract}

Keywords Suspension $\cdot$ Shear thickening $\cdot$ Cornstarch $\cdot$ Impact solidification

\section{Introduction}

Suspensions of cornstarch in water have become a shearthickening model system because they present continuous shear thickening, discontinuous shear thickening and shear jamming in a particularly clear manner $[2,16]$. Furthermore, the dramatic transition from a liquid-like to a solid-like material enables them to, for example, support a load [2], present cracks [17], and transmit a force between boundaries [10], features that distinctly set them apart from other liquids.

This article is part of the Topical Collection: In Memoriam of Robert P. Behringer.

Electronic supplementary material The online version of this article (https://doi.org/10.1007/s10035-019-0986-3) contains supplementary material, which is available to authorized users.

Devaraj van der Meer

d.vanderMeer@utwente.nl

1 Physics of Fluids Group, Faculty of Science and Technology, Mesa+ Institute, and J.M. Burgers Centre for Fluid Dynamics, University of Twente, 7500 AE Enschede, The Netherlands

2 Present Address: Saint-Gobain Research Provence, Cavaillon, France
Many efforts have been done to explain this peculiar behavior, including both steady-state $[2,16,18]$ and dynamic approaches $[6-8,12,15,19]$. However, there are phenomena that remain to be understood completely, in particular, the impact-activated solidification of cornstarch suspensions has proven to be highly non-trivial and multifaceted. It was shown that a localized jammed region grows ahead of the impactor $[10,19]$, with a velocity larger than that of the projectile $[6,15,19]$ and generates an added mass that however only partly accounts for the large decelerations that were measured. In fact, recent work from the Behringer group [9] provided evidence for the existence of two fronts, namely a fast pressure front followed by the slower, abovementioned solidification front. This was realized by replacing one of the side walls by a photo-elastic material, thus creating a sensitive device to measure pressure and pressure wave speeds.

Simultaneously, it has been observed that the velocity of a sphere settling in a deep bath of cornstarch suspension reaches an average terminal velocity around which it oscillates (bulk oscillations), while once the sphere approaches the bottom of the container, it undergoes what has been called stop-go cycles, in each of which the sphere comes to a complete stop and then re-accelerates again [7, 8]. Both observations were attributed to the building-up and subsequent relaxation of a solid plug. The complete stop during 

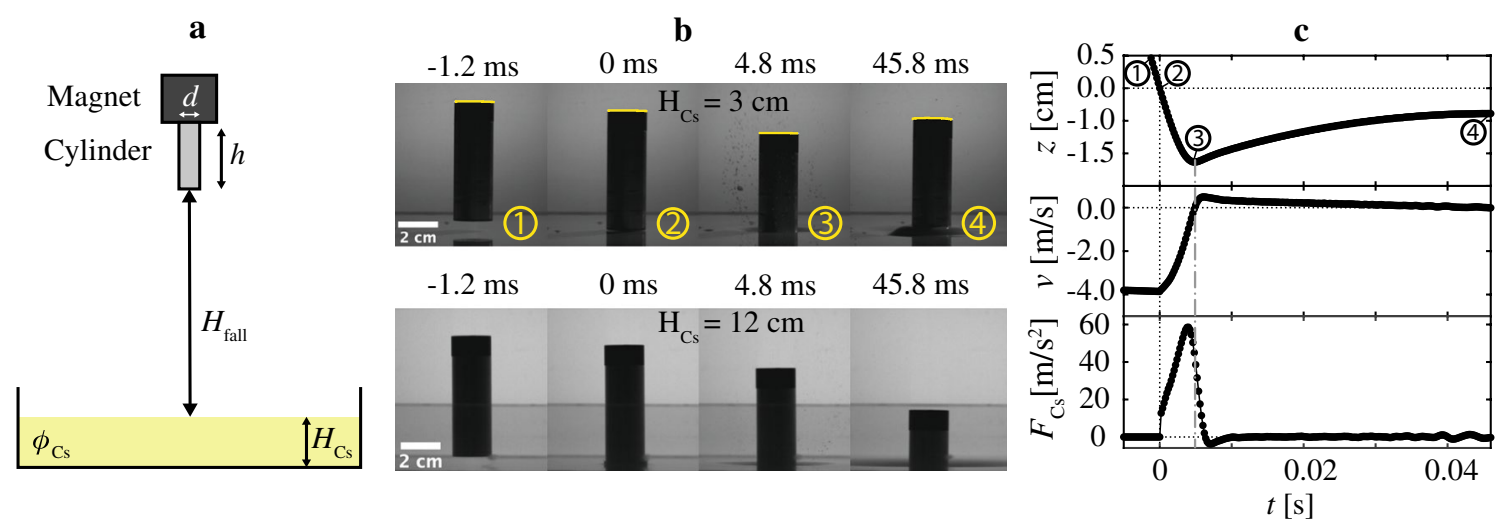

Fig. 1 a Sketch of the experimental set-up. b Two series of experimental snapshots for identical impacts with different cornstarch layer thickness: $H_{\mathrm{Cs}}=3.0 \mathrm{~cm}$ (top), and $H_{\mathrm{Cs}}=12.0 \mathrm{~cm}$ (bottom). In both cases, $M_{\text {cyl }}=49.6 \mathrm{~g}, \phi_{\mathrm{Cs}}=0.42$, and $H_{\text {fall }}=75 \mathrm{~cm}$ (see Online Resources 1 and 2 for movies of these experiments). $\mathbf{c}$ Time evolution of position $z$, velocity $v$, and force $F_{\mathrm{Cs}}$ (compensated for the cylinder weight) of the impacting cylinder, corresponding to the upper series

the stop-go cycles would then be caused by the interaction of this solid plug with the bottom boundary.

Direct interaction between the boundaries and the impactor is believed to occur only once the jammed front reached the boundaries, after which the deceleration is observed to be much larger than that caused by added mass [15]. It has been suggested that this large acceleration is the result of the compression of the jammed plug between the impactor and the boundaries $[1,11,13]$.

In this work we revisit the impact of a projectile on a water-cornstarch suspension. We focus our attention to shallow baths and high impact velocities, which in many cases causes the impactor to bounce. We explore the conditions that lead to bouncing and compare the time evolution of position, velocity and acceleration for cases with bouncing, i.e., where the impactor velocity changes sign, and cases in which the impactor position monotonously decreases until the object comes to rest.

As we will show below, in this way we are able to make a bridge between the studies that focused on the growth of the jammed front and those that deal with the settling of a sphere in a deep suspension.

The paper is structured as follows. In the next section, we will describe our experimental setup. Subsequently, we will present our results and discuss under which condition bouncing is observed. Turning to the trajectories of the impactor, we will discuss how these are affected by the effect of changing the depth of the suspension layer and how two very distinct regimes are observed, namely one that is dominated by a viscous response and the other by viscoelasticity (Sect. 3). We will conclude by discussing the different forces acting on the impactor in these two regimes. of snapshots. Note that $t=0$ corresponds to the moment of impact and the numbers correspond to the four snapshots in $b$. The dotted black lines indicate zero time, position, velocity, and force. The vertical dashed-dotted gray line marks the time at which the position minimum (zero velocity) is reached. Clearly, the maximum force is reached before this point in time

\section{Methods}

The experimental setup consists of an acrylic box (of height $15 \mathrm{~cm}$, and with a square bottom area of $30 \times 30 \mathrm{~cm}^{2}$ ) with transparent side walls, which contains the cornstarch suspension, as depicted in Fig. 1a. The depth of the suspension layer, $H_{\mathrm{Cs}}$, is varied from 1.0 to $12.0 \mathrm{~cm}$. The impactors are cylinders of fixed diameter $d=2 \mathrm{~cm}$ and height $h=6 \mathrm{~cm}$, with masses $M_{\text {cyl }}$ of $49.6 \mathrm{~g}$ and $129.9 \mathrm{~g}$ (consisting of aluminium and stainless steel, respectively). These were left to freely fall from different initial heights, $H_{\text {fall }}=0-150 \mathrm{~cm}$, resulting in velocities up to $5.4 \mathrm{~m} / \mathrm{s}$. To vary the falling height, the impactors were suspended from an electromagnet connected to a vertical translation stage. The impact event is recorded with a fast camera (Photron, FASTCAM Mini UX100) at 5000-6400 fps with a resolution of $65-68$ pixels $/ \mathrm{cm}$.

The suspensions are prepared using demineralized water and additive-free cooking cornstarch $(250 \mathrm{~g}$ sealed boxes, Maizena, Duryea). For every suspension layer depth $H_{\mathrm{Cs}}$ a new mixture was prepared. Before every single impact, the suspension was mixed thoroughly to avoid sedimentation. Four different cornstarch volume fractions $\phi_{\mathrm{Cs}}$ were tested, namely $0.38,0.40,0.42$, and 0.44 . We initially obtained the mass fraction of the suspension, then we converted it to volume fraction taking the density of cornstarch as: $\rho_{C s}=1,542 \mathrm{~kg} / \mathrm{m}^{3}[14]$ and the density of water as: $\rho_{w}=994 \mathrm{~kg} / \mathrm{m}^{3}$, measured with a density meter (DMA 35, Anton Paar). In this conversion, we have neglected the effect of the porosity of the cornstarch grains [5], which 


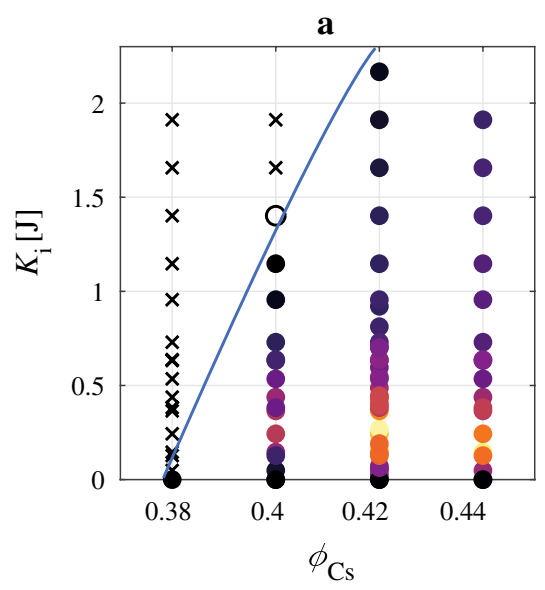

Fig. 2 Phase diagrams showing for which impact kinetic energy $K_{\mathrm{i}}$ the cylinder bounces versus a cornstarch volume fraction $\phi_{\mathrm{Cs}}$ (for constant $H_{\mathrm{Cs}}=3.0 \mathrm{~cm}$ ) and b suspension layer depth $H_{\mathrm{Cs}}$ (for constant $\left.\phi_{\mathrm{Cs}}=0.40\right)$. In these diagrams two different impactor masses are included. Circles represent bouncing events, where the color code represents the height the cylinder bounced (see color bar) and crosses represent cases where no bouncing occurs. Filled symbols indicate

may cause the true volume fractions to be slightly different from the reported ones, but will not affect our conclusions.

We tracked the position $z$ of the top of the cylinder versus time using a home-made MatLab code (see Fig. $1 b$ for an example of the detected top) and from it we obtained its velocity $v$ and acceleration $a$ by differentiating a piecewise 3rd degree polynomial fit to the position data. From the acceleration $a$, we obtained the force $F_{\mathrm{CS}}$ exerted by the suspension on the cylinder by subtracting the acceleration of gravity and multiplying by the mass of the cylinder. Typical time-evolution plots of the position $z$, velocity $v$, and force $F_{\mathrm{Cs}}$ are shown in Fig. 1c.

\section{Results and analysis}

The time-evolution curves plotted in Fig. 1c are reminiscent of the trajectories shown in [19], in the sense that both have a strong force peak just after impact. In our case, however the impactor is not only highly decelerated but even bounces back due to the shallowness of the suspension layer. The rebound occurs at about $1.5 \mathrm{~cm}$ above the bottom of the container $\left(H_{\mathrm{Cs}}=3.0 \mathrm{~cm}\right)$, which means that there is no direct contact between the cylinder and the container, but suggests a solid-like stress transmission between them [10]. When we increase the suspension layer depth, the impactor continues to sink (Fig. 1b, bottom snapshots).

To map out the bouncing behavior of the suspension layer, we systematically changed the layer depth $H_{\mathrm{Cs}}$ and volume fraction $\phi_{\mathrm{Cs}}$ and for each case impacted it with different masses and velocities to explore under which conditions

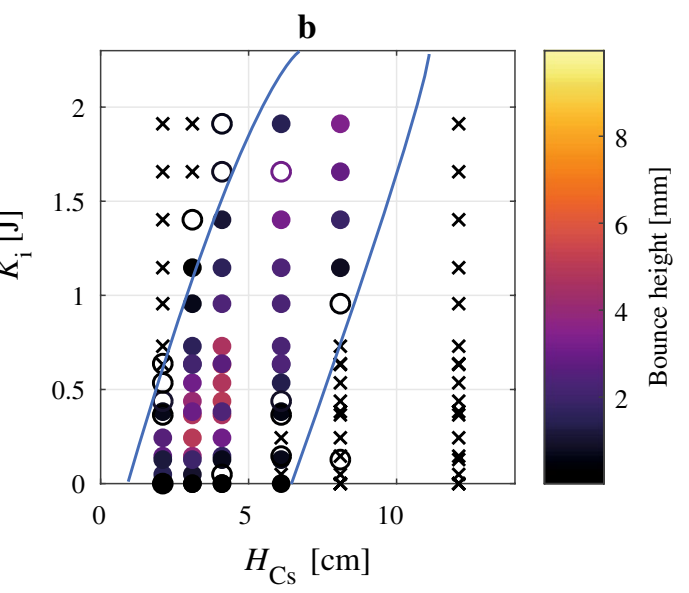

cases where bouncing is always observed, whereas for open symbols bouncing occurred in a subset of repetitions only. The blue lines are guides to the eye, qualitatively separating the region where bouncing occurs from other regions. Note that the lowest kinetic energy of impact is equal to zero, where we released the cylinder from the surface of the suspension (color figure online)

bouncing was observed. The result is summarized in Fig. 2, where we plot two phase diagrams showing for which conditions bouncing occurs as a function of $\phi_{\mathrm{Cs}}$ and $H_{\mathrm{Cs}}$. We combined the effects of cylinder mass and impact velocity by plotting the kinetic energy at impact, $K_{\mathrm{i}}=M_{\text {cyl }} g H_{\text {fall }}{ }^{1}$. This allowed us to match the results from different cylinder masses for $\phi_{\mathrm{Cs}} \geq 0.4$, as shown in Fig. $2 \mathrm{a}$.

Figure $2 \mathrm{a}$ shows the effect of solid fraction on bouncing (at a constant layer depth $H_{\mathrm{Cs}}=3.0 \mathrm{~cm}$ ). For $\phi_{\mathrm{Cs}}<0.4$ (50.8 wt \%), excepting $K_{\mathrm{i}}=0$, the impactor doesn't bounce, in accordance with the observations of Crawford et al. [3], where the kinetic energy of impact was large and their impactor simply sank at low mass fraction. Moreover, while at $\phi_{\mathrm{Cs}}=0.4$ the impactor bounces for low $K_{\mathrm{i}}$, for high impact kinetic energies the cylinder travels all the way to the bottom to either completely stop or to dramatically bounce due to direct contact with the bottom wall.

The effect of the suspension layer thickness is shown in Fig. $2 \mathrm{~b}$ (for constant $\phi_{\mathrm{Cs}}=0.4$ ). For the deepest layer used, no bouncing is observed at any kinetic energy of impact (as long as we can continue to track the cylinder), while bouncing happens for lower layer depths. However, for $H_{\mathrm{Cs}}=8.0 \mathrm{~cm}$, bouncing is observed only for sufficiently high impact kinetic energies, whereas lower $K_{\mathrm{i}}$ present monotonous sinking of the impactor. For shallower cases,

\footnotetext{
${ }^{1}$ Note that non-dimensionalizing $K_{\mathrm{i}}$ with some property of the cylinder (such as the associated potential energy $M_{\text {cyl }} g h$ ) does not collapse the data, which suggests that some viscous or elastic property of the cornstarch layer may deliver proper non-dimensionalization.
} 

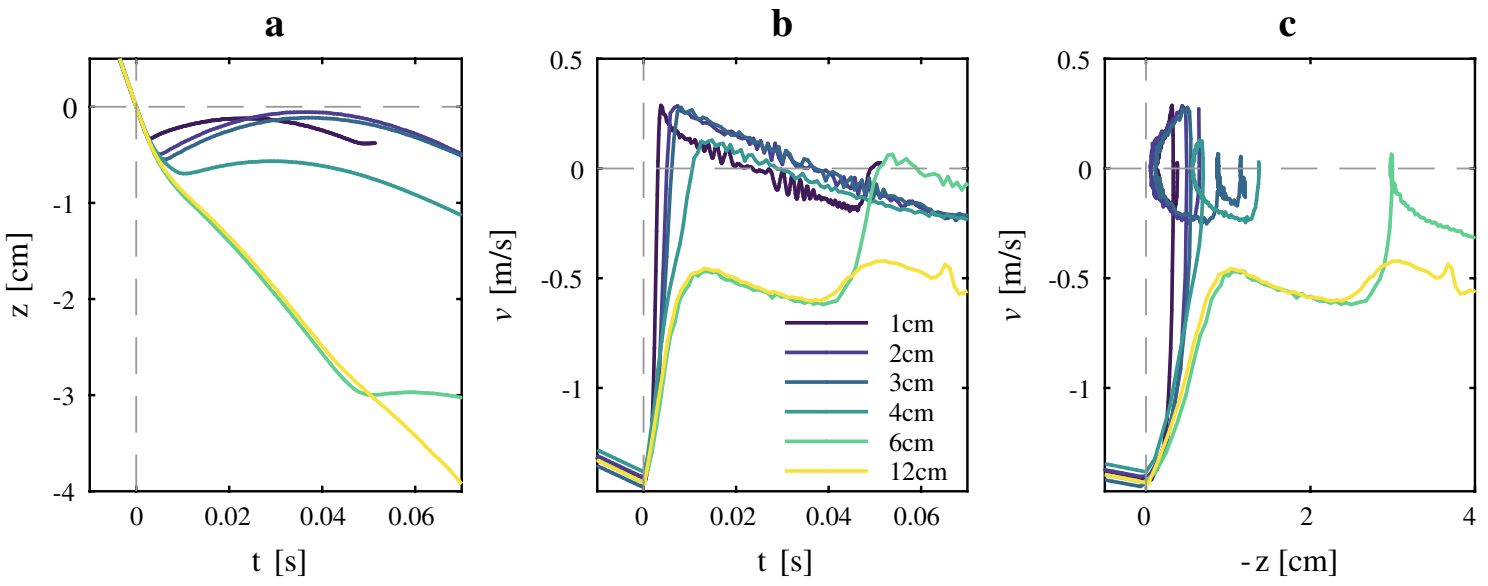

Fig. 3 Cylinder position and velocity for different layer depths $H_{\mathrm{Cs}}$ between 1.0 and $12.0 \mathrm{~cm}$ (see legend), keeping all other parameters fixed to $M_{\text {cyl }}=49.6 \mathrm{~g}, \phi_{\mathrm{Cs}}=0.42$, and $H_{\text {fall }}=10.0 \mathrm{~cm}$. a Position

$z$ as a function of time $t, \mathbf{b}$ velocity $v$ as a function of time $t$, and $\mathbf{c}$ velocity $v$ as a function of the distance $-z$ to the surface of the cornstarch layer

even towards zero impact kinetic energy a slight rebound was recorded. As described above, for the shallowest cases there is a maximum kinetic energy beyond which the impactor doesn't bounce anymore.

The color code in Fig. 2 represents the height the cylinder bounced, showing that there is a maximum rebound height for intermediate kinetic energies. Moreover, close to the boundaries of the bouncing regions the rebound height becomes small, suggesting a smooth transition between both regions. Clearly, the impact kinetic energy plays a role in determining if bouncing occurs or not. Part of the impact energy is dissipated due to viscous effects (although other effects like friction or cracking may also contribute) and another part is transferred into the solidified added mass in front of the impactor ${ }^{2}$. Finally once the front has reached the boundaries, the remaining energy may largely be transferred into plastic and elastic deformation of the solidified plug and may partly be recovered for bouncing. Note that the bounce is only in the order of a few millimetres as compared to tens of centimetres of $H_{\text {fall }}$, which implies that only a tiny fraction of the impact kinetic energy is recovered during rebound.

In addition, the increase of the upper boundary of the bouncing region with increasing $\phi_{\mathrm{Cs}}$ and $H_{\mathrm{Cs}}$ suggests that for too dilute or shallow cases, the suspension layer cannot dissipate enough energy to stop the cylinder before reaching the bottom of the container, as also suggested by Roché et al. [17], which implies that as the solid fraction or the layer depth increase, the suspension obtains a larger capacity for dissipating and storing energy.

\footnotetext{
${ }^{2}$ In [6], a minimum impact velocity was required to enable detection of the propagation of any front.
}

Now, what causes this peculiar behavior of the cornstarch suspension layer? To obtain additional insight we now turn to measurements with the same impact velocity (i.e., the same $H_{\text {fall }}$ ) but varying cornstarch layer depth $H_{\mathrm{Cs}}$. In Fig. 3a we plot the typical time evolution of the vertical position $z$ of the cylinder for different suspension depths, fixing the other parameters to $M_{\text {cyl }}=49.6 \mathrm{~g}, \phi_{\mathrm{Cs}}=0.42$, and $H_{\text {fall }}=10 \mathrm{~cm}$. Initially, all individual experiments appear to follow the same master curve but eventually deviate, and reach a minimum $z_{\text {min }}$ after which the postion increases again (i.e., the cylinder bounces). The deeper the cornstarch layer is, the longer the the position follows the master curve. In the deepest case, $H_{\mathrm{Cs}}=12.0 \mathrm{~cm}$, no bouncing is observed. This is consistent with our earlier observations: Note that we travel a horizontal path through a phase diagram similar to Fig. 2b, where for small $H_{\mathrm{Cs}}$ bouncing is observed, whereas for large $H_{\mathrm{Cs}}$ the cylinders sink continuously.

We interpret the time at which the deviation from the master curve occurs as the moment the solidified front has reached the bottom of the container, as was shown in $[1,11]$. This is consistent with the observation that shallower layer depths present an earlier time of deviation, since there the jammed front has to grow over a smaller distance, before it reaches the bottom of the container. It is tempting to check whether the deviation always occurs at the same distance from the bottom. We did, and in fact this is not the case: The distance above the bottom increases with the layer depth, which is again consistent with the growth of a cornstarch plug below the cylinder in time.

Figure $3 b, c$ show the cylinder velocity versus time and position, respectively. From these figures two very distinct behaviors appear: One is observed for large layer depths $H_{\mathrm{Cs}}$ only, where the velocity reaches a quasi-terminal velocity with behavior that indicates the bulk oscillations described 
Fig. 4 Force exerted by the cornstarch suspension on the cylinder as a function of $\mathbf{a}$ the distance $-z$ to the surface of the cornstarch layer and $\mathbf{b}$ the velocity $v$, for the same experiments that were presented in Fig. 3, and using the same legend. The inset in a shows a zoomed-in region for the first $0.8 \mathrm{~cm}$. The arrow in $\mathbf{b}$ indicates the direction of increasing time a

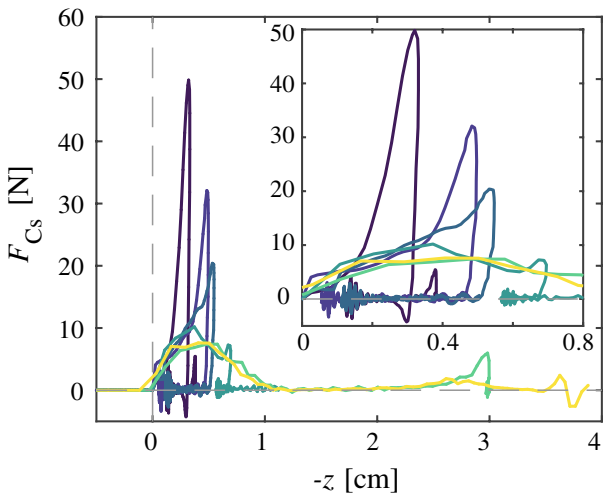

b

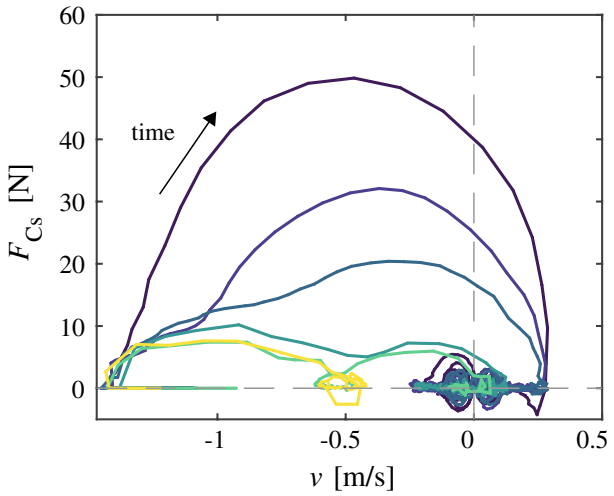

in [7], and another where the velocity suddenly and rapidly goes to zero and becomes positive.

Interestingly, the difference in the times at which deviation occurs between $H_{\mathrm{Cs}}=4.0 \mathrm{~cm}$ and $6.0 \mathrm{~cm}$, is much larger than the deviation-time difference between the cases of $H_{\mathrm{Cs}}=2.0$ and $4.0 \mathrm{~cm}$, which is in contradiction with the findings by [19], where the time for the front to reach the bottom scales close to linearly with the height of the suspension. The reason may lie in the following observation from Fig. 3b: It can be seen that for $H_{\mathrm{Cs}} \leq 4.0 \mathrm{~cm}$ the deviation happens before the velocity has reached the quasi-terminal velocity, while for the $H_{\mathrm{Cs}} \geq 6.0 \mathrm{~cm}$ case it happens afterwards, but just before the second oscillation. This indicates that whereas for small layer depths the cornstarch plug may hit the bottom during its growing phase (leading to the linear behavior of [19]), for large layer depths the plug is in a (quasi-)steady state between growing and melting, corresponding to the development of a terminal velocity.

It is worth noting that a rebound may be followed by a second and even a third bounce or stopping event, reminiscent of the stop-go cycles reported in [7]. This can be best appreciated in Fig. $3 \mathrm{c}$, where, e.g., for $H_{\mathrm{Cs}}=3.0 \mathrm{~cm}$, one observes after the first rebound (signalled by $v=0$ ) at a depth of $\approx 0.6 \mathrm{~cm}$, a second and third event at depths of 0.9 and $1.2 \mathrm{~cm}$, respectively. It should be noted that in our case the impactor is not completely immersed so after bouncing there may be a period of ballistic free-fall, whereas during the stop-go cycles for the fully immersed objects reported in [7] a drag force is always present.

In Online Resource 3, we show that when keeping the layer depth $H_{\mathrm{Cs}}$ fixed and increasing the fall height $H_{\text {fall }}$ (and thus the impact velocity), the time at which the curve starts to deviate reduces. This is not surprising, given that at a higher impact velocity the jammed front is expected to grow faster [6].

In the final part of this section we will concentrate on the force $F_{\mathrm{CS}}$ that the impactor experiences inside the suspension, which we obtain by subtracting the gravitational acceleration $g$ from the measured acceleration $a$ and subsequently multiplying with the cylinder mass $M_{\text {cyl }}$. In Fig. 4 we plot this force as a function of the distance $-z$ of the impactor to the surface and its vertical velocity $v$.

Looking at Fig. $4 \mathrm{a}$, where $F_{\mathrm{Cs}}$ is plotted versus $-z$, we retrieve the two regimes found in Fig. 3: There is an approximately linear master curve (inset Fig. 4a), where a strong force peak is observed as soon as the cylinder starts to bounce. We can now interpret these two behaviors as a regime, corresponding to the master curve, that is dominated by viscous and added mass forces [19] and a regime that is dominated by viscoelastic forces. The height of this peak decreases with increasing suspension layer depth. This stands to reason, since in a deeper bath there is a larger time span in which viscous dissipation can occur, and in addition, a larger plug and therefore a larger added mass is created. Thus, by the time the front interacts with the boundary, it has less kinetic energy to deform the plug ${ }^{3}$.

Turning to Fig. $4 \mathrm{~b}$, in which $F_{\mathrm{Cs}}$ is plotted as a function of velocity $v$, we see that the curves for $H_{\mathrm{Cs}} \leq 3.0 \mathrm{~cm}$ that are very close in $(v, z)$-space (Fig. 3$)$ are very far apart in $\left(F_{\mathrm{Cs}}, v\right)$ -space, whereas the other curves $\left(H_{\mathrm{Cs}} \geq 4.0 \mathrm{~cm}\right)$ which are very different in Fig 3, are relatively close in Fig. 4b. In fact, the curves for $H_{C s}=4.0 \mathrm{~cm}$ and $H_{C s}=6.0 \mathrm{~cm}$ almost overlap.

Once the bouncing cases start to deviate from the master curve, the dominant force should come from the compression of the jammed plug between the impactor and the boundary. If the compression would be purely elastic however, the force should be the same linear function of position on the increasing and decreasing path, with a maximum at the moment the impactor stops. Clearly, from Fig. 4a it appears that the force doesn't follow the same path before and after reaching the maximum, instead it decreases with a steeper slope. A closer look at the decreasing section of the force curve (inset of Fig. 4a) reveals that after reaching the

\footnotetext{
3 This is analogous to the observation that less cracks appear in deeper baths of cornstarch [17].
} 
maximum force, the impactor continues to sink a very small distance before it bounces back. This is also clear from the $F_{\mathrm{Cs}}$ versus $v$ plot (Fig. $4 \mathrm{~b}$ ) where the maximum force does not occur for $v=0$, but for slightly negative $v$, i.e., the cylinder will continue to sink after reaching the maximum force.

This kind of hysteretic behavior of the force as a function of position resembles that of a viscoelastic solid. To obtain some quantitative order of magnitude estimates for the elastic and viscous properties of the cornstarch suspension, we therefore used the simplest linear, viscoelastic solid model available, namely the Kelvin-Voigt solid. This model consists of a dashpot and a spring in parallel, where the jammed plug can be thought of as a series of parallel springs immersed in a liquid (water). We solved the resulting equation of motion $M_{\text {cyl }} \ddot{z}+b \dot{z}+k z=0$, where $b$ and $k$ are a drag and a spring constant, respectively, with initial conditions $z\left(t_{\mathrm{d}}\right)=0$ and $\dot{z}\left(t_{\mathrm{d}}\right)=v_{\mathrm{dev}}$, where $v_{\text {dev }}$ is the velocity of the cylinder at the moment $t=t_{\mathrm{d}}$ the curves start to deviate from the master curve. We fitted only the deviatoric part of our experimental curves, for $\phi_{C s}=0.40,0.42$ and for $H_{\mathrm{Cs}}=1.0,2.0,3.0 \mathrm{~cm}$ (a more detailed explanation of the fit can be found in the Online Resource 4). For the two values of $\phi_{C s}$, this resulted in two estimates for the spring constant: $k_{0.40}=8 \pm 3 \mathrm{kN} / \mathrm{m}$ and $k_{0.42}=25 \pm 9 \mathrm{kN} / \mathrm{m}$, and two estimates of the drag coefficient: $b_{0.40}=7 \pm 2 \mathrm{~kg} / \mathrm{s}$ and $b_{0.42}=15 \pm 6 \mathrm{~kg} / \mathrm{s}$, where the error intervals represent one standard deviation. Furthermore, by looking at the position at which the deviation occurred, we estimated the initial size of the plug, $L_{0}$, and with the base area of the impactor, $A$, we estimated the Young modulus $E \approx k L_{0} / A$ as: $E_{0.40}=0.3 \mathrm{MPa}$ and $E_{0.42}=0.8 \mathrm{MPa}$. We emphasize that these should be considered as order of magnitude estimates only, due to the uncertainty in the exact moment the plug comes in contact with the boundaries and the fact that the Kelvin-Voigt model is a very crude approximation for describing the jammed plug. We should note that once the plug is in contact with the boundary and it starts to be deformed it is possible that some changes in the volume fraction occur, therefore the drag coefficient might not be the same for the whole process. Finally, we fitted the model by Maharjan et al. [11] to the force curves after deviation but before the force became maximum and we obtained for the elastic modulus $E_{0.40}=0.3 \pm 0.1 \mathrm{MPa}$ and $E_{0.42}=0.6 \pm 0.3 \mathrm{MPa}$, which are very similar to those obtained with the Kelvin-Voigt model.

\section{Conclusion}

In conclusion, we have revisited the impact solidification of a cornstarch-water suspension for different suspension volume fractions $\left(\phi_{\mathrm{Cs}}\right)$ and cornstarch layer depths $\left(H_{\mathrm{Cs}}\right)$, as well as for different impactor masses and velocities. We found that for large depths, a minimum impact kinetic energy is necessary for the impactor to bounce. However, if the kinetic energy is too large and the suspension bath too shallow or too dilute, then no rebound is observed. Therefore, just as in [13] a minimum layer depth was found for the added mass model to be able to allow for an adult to run on top of a cornstarch pool, here we found that for a given kinetic energy, there is a minimum layer depth above which the compression of the solidified plug is able to cause the impactor to jump.

We found that the time-evolution curves for different layer depths follow the same master trajectory but eventually deviate, with the deviation time increasing with the depth of the suspension and decreasing with the impact velocity. Additionally, if the layer depth is large enough to allow the impactor to reach a quasi-terminal velocity [7], then the deviation time increases, presumably due to melting of the solidified front. In this way, we connect the literature that deals with the settling of an object in a deep cornstarch layer $[7,8,14]$ with the work concentrating on (high-speed) impact on a cornstarch layer $[6,11,13,19]$.

Finally, we observed that the force response for the cases with bounce resembles that of a viscoelastic solid and were able to provide an order of magnitude estimate of its properties. We want to stress that models like the Kelvin-Voigt model used here are crude approximations to a complex reality that is in need of substantial research to capture the complex processes during the compression of the plug and just before bouncing.

Close to publication we became aware of a similar work studying the impact of a sphere on a thin layer of cornstarch suspensions by Egawa and Katsuragi [4]. The results of that paper, where the focus lies on interpretation of the results using a Kelvin-Voigt model, appear to be in accordance with our results.

Acknowledgements The authors thank M. van den Berg for collecting part of the data. This work is part of a research program of the Foundation for Fundamental Research on Matter (FOM), which is financially supported by the Netherlands Organisation for Scientific Research (NWO). R.A.L.C. is supported by CONACYT (Scholarship Number 411297) and A.P. by a FOM/v postdoctoral grant.

Data availability The datasets generated during and/or analysed during the current study are available from the corresponding author on reasonable request.

\section{Compliance with ethical standards}

Conflict of interest The authors declare that they have no conflict of interest.

Open Access This article is licensed under a Creative Commons Attribution 4.0 International License, which permits use, sharing, adaptation, distribution and reproduction in any medium or format, as long as you give appropriate credit to the original author(s) and the source, provide a link to the Creative Commons licence, and indicate if changes 
were made. The images or other third party material in this article are included in the article's Creative Commons licence, unless indicated otherwise in a credit line to the material. If material is not included in the article's Creative Commons licence and your intended use is not permitted by statutory regulation or exceeds the permitted use, you will need to obtain permission directly from the copyright holder. To view a copy of this licence, visit http://creativecommons.org/licenses/by/4.0/.

\section{References}

1. Allen, B., Sokol, B., Mukhopadhyay, S., Maharjan, R., Brown, E.: System-spanning dynamically jammed region in response to impact of cornstarch and water suspensions. Phys. Rev. E 97, 052603 (2018). https://doi.org/10.1103/PhysRevE.97.052603

2. Brown, E., Jaeger, H.M.: Shear thickening in concentrated suspensions: phenomenology, mechanisms and relations to jamming. Rep. Progr. Phys. 77(4), 046602 (2014). https://doi. org/10.1088/0034-4885/77/4/046602

3. Crawford, N.C., Popp, L.B., Johns, K.E., Caire, L.M., Peterson, B.N., Liberatore, M.W.: Shear thickening of corn starch suspensions: does concentration matter? J. Colloid Interface Sci. 396, 83-89 (2013). https://doi.org/10.1016/j.jcis.2013.01.024

4. Egawa, K., Katsuragi, H.: Bouncing of a projectile impacting a dense potato-starch suspension layer. Phys. Fluids 31(5), 053304 (2019). https://doi.org/10.1063/1.5095678

5. Han, E., Van Ha, N., Jaeger, H.M.: Measuring the porosity and compressibility of liquid-suspended porous particles using ultrasound. Soft Matter 13, 3506-3513 (2017). https://doi.org/10.1039/ C7SM00182G

6. Han, E., Peters, I.R., Jaeger, H.M.: High-speed ultrasound imaging in dense suspensions reveals impact-activated solidification due to dynamic shear jamming. Nat. Commun. (2016). https://doi. org/10.1038/ncomms12243

7. von Kann, S., Snoeijer, J.H., Lohse, D., van der Meer, D. Nonmonotonic settling of a sphere in a cornstarch suspension. Phys. Rev. E 84, 060401 (2011). https://doi.org/10.1103/PhysR evE.84.060401

8. von Kann, S., Snoeijer, J.H., van der Meer, D.: Velocity oscillations and stop-go cycles: the trajectory of an object settling in a cornstarch suspension. Phys. Rev. E 87, 042301 (2013). https:// doi.org/10.1103/PhysRevE.87.042301

9. Lim, M.X., Barés, J., Zheng, H., Behringer, R.P.: Force and mass dynamics in non-newtonian suspensions. Phys. Rev. Lett. 119(18), 184501 (2017). https://doi.org/10.1103/PhysRevLett.119.184501
10. Liu, B., Shelley, M., Zhang, J.: Focused force transmission through an aqueous suspension of granules. Phys. Rev. Lett. 105, 188301 (2010). https://doi.org/10.1103/PhysRevLett.105.188301

11. Maharjan, R., Mukhopadhyay, S., Allen, B., Storz, T., Brown, E.: Constitutive relation for the system-spanning dynamically jammed region in response to impact of cornstarch and water suspensions. Phys. Rev. E 97, 052602 (2018). https://doi.org/10.1103/PhysR evE.97.052602

12. Merkt, F.S., Deegan, R.D., Goldman, D.I., Rericha, E.C., Swinney, H.L.: Persistent holes in a fluid. Phys. Rev. Lett. 92, 184501 (2004). https://doi.org/10.1103/PhysRevLett.92.184501

13. Mukhopadhyay, S., Allen, B., Brown, E.: Testing constitutive relations by running and walking on cornstarch and water suspensions. Phys. Rev. E 97, 052604 (2018). https://doi.org/10.1103/ PhysRevE.97.052604

14. Oyarte Gálvez, L., de Beer, S., van der Meer, D., Pons, A.: Dramatic effect of fluid chemistry on cornstarch suspensions: linking particle interactions to macroscopic rheology. Phys. Rev. E 95, 030602 (2017). https://doi.org/10.1103/PhysRevE.95.030602

15. Peters, I.R., Jaeger, H.M.: Quasi-2D dynamic jamming in cornstarch suspensions: visualization and force measurements. Soft Matter 10, 6564-6570 (2014). https://doi.org/10.1039/C4SM0 0864B

16. Peters, I.R., Majumdar, S., Jaeger, H.M.: Direct observation of dynamic shear jamming in dense suspensions. Nature (2016). https://doi.org/10.1038/nature17167

17. Roché, M., Myftiu, E., Johnston, M.C., Kim, P., Stone, H.A.: Dynamic fracture of nonglassy suspensions. Phys. Rev. Lett. 110, 148304 (2013). https://doi.org/10.1103/PhysRevLett.110.148304

18. Wagner, N.J., Brady, J.F.: Shear thickening in colloidaldispersions. Phys. Today 62(10), 27-32 (2009). https://doi. org/10.1063/1.3248476

19. Waitukaitis, S.R., Jaeger, H.M.: Impact-activated solidification of dense suspensions via dynamic jamming fronts. Nature 487(7406), 205-209 (2012). https://doi.org/10.1038/nature11187

Publisher's Note Springer Nature remains neutral with regard to jurisdictional claims in published maps and institutional affiliations. 